\title{
Development of neurophysiological aspects of the spinal cord during the past ten years
}

\author{
M R Dimitrijevic MD DSc
}

Baylor College of Medicine, Division of Restorative Neurology and Human

Neurobiology, Houston, Texas, USA.

\begin{abstract}
Although neurophysiological knowledge of the spinal cord has advanced significantly, the appearance of the spinal cord has not changed. The spinal cord is still an elongated, cylindrical portion of the central nervous system, which consists of peripheral white matter with propriospinal, long ascending and descending pathways surrounding the central gray matter. It has been shown that there are at least 3 systems of motor pathways originating in the brain and ending in the spinal cord: the system from the cerebral cortex, the brain stem and the 'gain-setting' system, which are under limbic control. ${ }^{1}$ The latter system has neurons in the nucleus coeruleus, the subcoeruleus and raphe (midline) nuclei of the lower brain stem, including the ventral reticular formation with projection to the posterior, intermedial and anterior portions of the spind cord gray matter. These pathways are diffusely organized and their role can be seen as a 'gain-setting system', activated during excessive motivational responses.
\end{abstract}

The distribution pattern of the descending system terminals has been extensively studied in cat, macaque monkeys and also in humans, and we know more about descending axon connections and their distribution, particularly within the dorsolateral, ventromedial part of the intermediate zone of spinal cord interneurons. This distribution pattern in afferent connections with spinal interneurons is relevant for the understanding of supraspinal and spinal connectivity. It can be speculated that a different set of interneurons in different parts of the intermediate zone possesses the same characteristics as some of the descending pathways which terminate on them. According to Kuypers, ${ }^{2}$ the interneurons of the ventral medial part of the intermediate zone represent a diffuse system, while those in dorsal and lateral parts represent a focused system. Therefore, certain individual interneurons can maintain widespread connections with a variety of motoneurons in a large portion of the spinal cord, while those in other areas are rather restricted to a particular part of the interneurons (Fig 1).

The spinal cord is a 'lower motor center' and not only a reflex center of proprio- and exteroceptive reflexes. In the past, spinal interneurons were mainly seen as a network of excitatory and inhibitory neurons involved in changing peripheral input. Thus, until recently, it was not certain whether there were 2 separate subgroups of spinal interneurons, one involved in spinal reflex activity and another in brain control of the 'lower spinal motor center'. In the last 10 years, researchers provided definite evidence that the spinal interneuronal system is not divided into 2 separate subgroups, but is an entity that integrates both functions in order to produce harmonious movements. ${ }^{3}$ The premotoneuronal integration center is the last link of various peripheral and brain pathways, and the convergence of primary afferents and of fibers of central origin upon interneurons appears to be the rule. ${ }^{3}$ Spinal interneurons, therefore, can adjust the degree of inhibition of various flexors and extensors so that those muscles can either contract alternately or simultaneously.

These 2 new concepts (1) somatotopic organization of the brain's descending system to the intermediate zone of spinal interneurons, and (2) premotoneuronal spinal motor centers play an important role in studies of the neuronal system of movement control via interneurons. ${ }^{4,5}$ Thus, if neurophysiology of the spinal motor system 


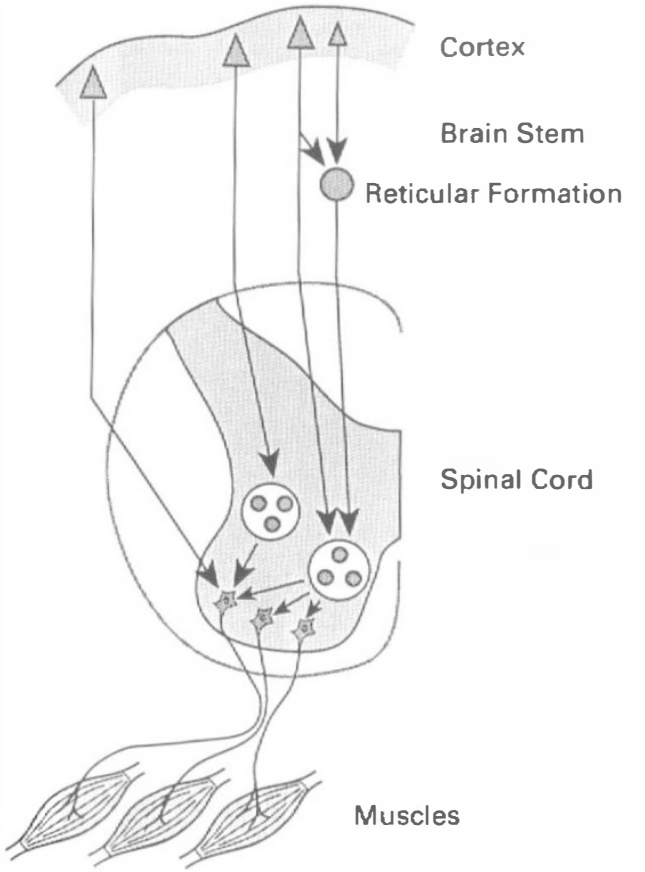

Figure 1 Schematic drawing of pattern distribution of cortico and bulbospinal pathways on interneurons of intermediate zone of the spinal cord's gray matter.

in the 60 s and 70 s focused on identifying reflex pathways from muscle spindle afferents, golgi tendon organs, flexion reflex, etc, in healthy subjects and humans with spinal cord injuries or other neurological disorders, in the 80s the neurophysiology of the spinal cord progressed in the analysis of brain influence on spinal reflex activities. In the past 10 years, it became clear that the brain is not only involved in the control of spinal motor activity but also in the control to set and anticipate motor events well before they become manifest. Thus, spinal cord motor activity is influenced by the brain to anticipate, to set the coming events and, finally, to execute tuned and well adjusted movements (Fig 3 ).

In cases of spinal cord injury, particularly one that completely or incompletely divides the 'upper brain' and 'lower spinal cord' motor centers, new motor control alternatives can be generated as well as new features of spinal reflex activity. The segmental reflex, intersegmental spinal and propriospinal systems will generate different

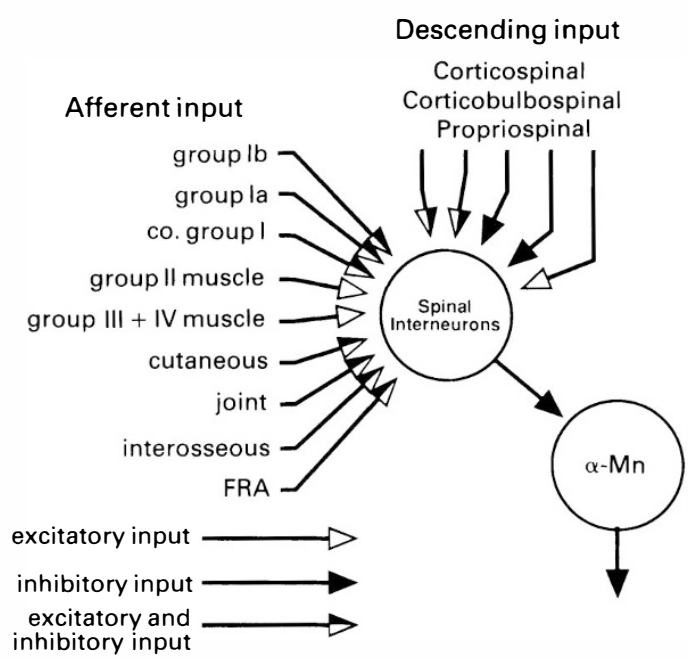

Figure 2 Simplified scheme illustrating the concept of convergence of segmental and suprasegmental volleys on the interneuron, before the activation of spinal motor cells.

motor control. Therefore, contemporary neurophysiology requires that after examining independently suprasegmental and segmental motor activity of the spinal cord of humans with spinal cord disorders, we must also evaluate how segmental motor activity depends upon suprasegmental influence. Moreover, the evaluation of the degree to which supraspinal control can modify reflex activity is imperative in the neurological evaluation of spinal cord motor functions. Schomburg $^{3}$ in his review on the spinal sensory motor system and its supraspinal control pointed out that the notion that spinal reflex activity and brain motor control are integrated in a converging interneuronal system, the premotoneuronal center, is not new and was already described by Förster in 1879 . What is different is that we now have the technology to study supraspinal and spinal cord motor activities in animals as well as in humans.

During the 80 s we acquired another important method to study brain and spinal cord motor activity interactions by transcranial motor cortex stimulation in humans. ${ }^{6}$ For such a purpose we have available high voltage electrical and magnetic stimulators. It has been shown that the conduction time and velocity of the fastest fibers of the cortico-motoneuronal system can be 


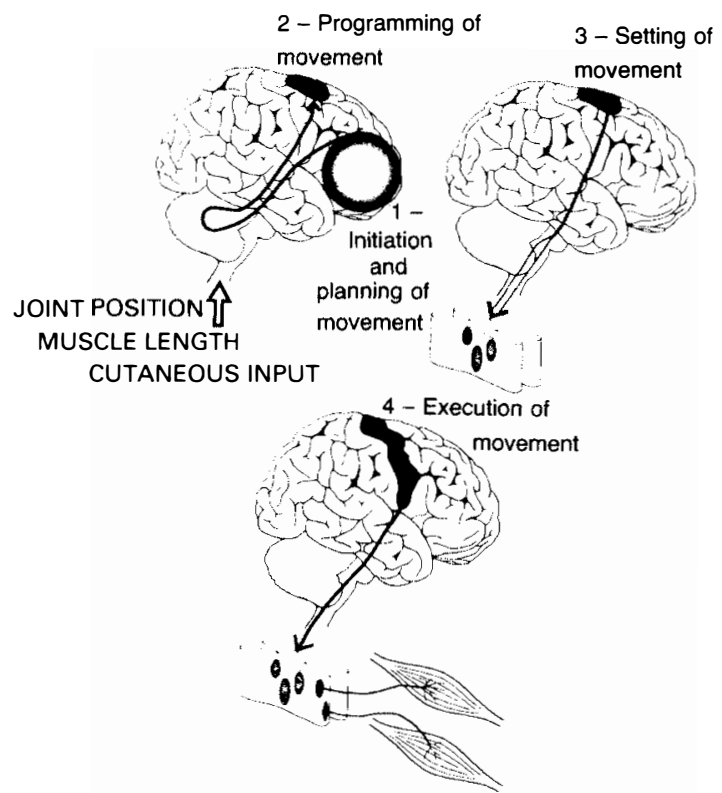

Figure 3 Schematic illustration of deciding, setting and executing movement and brain, spinal cord functional interaction.

measured and even that it is possible to stimulate the descending pathways at the level of the brain stem and probably also of the spinal cord.

And what about the sensory spinal cord system? Thanks to the involvement of the spinal cord gray matter in pain, progress was made in the neurophysiology of sensory processing of afferent input, relevant mechanisms to peripheral and central pain.? Moreover, there are also experimental animal and clinical studies providing information that sensory input and processing can be modified by the brain's descending influence. Thus, examination of sensory disturbances reveals that these are due not only to the impairment of spinal sensory mechanisms but also to altered brain influence. Let us also mention the expansion of monitoring techniques for the assessment of spinal cord functions during surgical interventions on the spinal cord, ${ }^{8}$ and the early development of an electrophysiological tool that can be placed in the epidural space for the electrophysiological and pharmacological evaluation of spinal cord functions in humans with acute and chronic cord neurological conditions. ${ }^{9}$
Let us ask ourselves what we can expect in the coming years. The impact of neurophysiology on research and the clinical practice of spinal cord disorders will continue and will be influenced by the development of neurobiology of the nervous system and by newly developing possibilities of applying biological materials, substances and implants to repair spinal cord functions. The advances in the development of reconstructive and restorative procedures for impaired spinal cord functions will demand extensive neurophysiological evaluation before intervention and monitoring of spinal cord functions during and after intervention. Moreover, if pharmacological interventions continue being more and more successful in minimizing the effects of injury or disease on spinal cord functions, then we shall grow more dependent upon neurophysiological methods while restoring the functional capabilities of the minimally or moderately affected spinal cord. Once we interact with specific regional and segmental spinal cord structures and their isolated or integrated functions, we will be dependent not only upon clinical and radiological evaluations, biochemistry and molecular bi- 
ology, but also on the electrophysiology of cellular and circuit functions as well as subclinical findings.

Hopefully, we shall once more be interested in examining the nature of spinal shock. At present, it is possible that we are classifying under spinal shock very different categories of spinal cord injuries that can result from central as well as peripheral nervous system mechanisms.

When reading the articles of the first 30 years of the journal Paraplegia, the progress that followed the pioneering work of Sir Ludwig Gutmann becomes obvious. Naturally, we can expect that neurophysiology will continue to be a part of the practice of physicians taking care of paraplegics. In addition, new methods for the assessment of functions of the spinal cord and progress in neurobiology will influence the newly established clinical practice for the prevention of the sequelae of spinal cord injuries and neurobiological interventions for the restoration of impaired functions.

\section{References}

1 Kuypers HGJM (1987) Some aspects of the organization of the output of the motor cortex. Motor Areas of the Cerebral Cortex, CIBA Foundation Symposium 132. John Wiley \& Sons, Chichester: 63-82.

2 Kuypers HGJM (1989) Motor system organization. In: Adelman G, ed. Encyclopedia of Neuroscience, Suppl 1. Birkhauser, Boston: 107-110.

3 Schomburg ED (1990) Spinal sensorimotor systems and their supraspinal control. Neurosci Res 7: 265-340.

4 Jankowska E (1989) A neuronal system of movement control via muscle spindle secondaries. In: Allum JHJ, Hulliger M, eds. Progress in Brain Research, Vol 80. Elsevier Science Publishers BV, Amsterdam: 299-303.

5 Pierrot-deseilligny P (1989) Peripheral and descending control of neurones mediating non-monosynaptic Ia excitation to motoneurones: a presumed propriospinal system in man. In: Allum JHJ, Hulliger M, eds. Progress in Brain Research, Vol 80. Elsevier Science Publishers BV, Amsterdam: 305-314.

6 Rothwell JC, Thompson PD, Day BL, et al (1987) Motor cortex stimulation in intact man. Brain 110: 1173-1190.

7 Dimitrijevic MR, Wall PD, Lindblom U, eds (1990) Recent Achievements in Restorative Neurology: Altered Sensation and Pain, Vol 3. Karker, Basel.

8 Ducker TB, Brown RH, eds (1988) Neurophysiology and Standards of Spinal Cord Monitoring. SpringerVerlag, New York.

9 Dimitrijevic MR, Sherwood AM (1982) A system for epidural spinal cord evaluation. Appl Neurophsiol 45: 465-467. 